\title{
Unmanned Aerial System for Antenna Measurement (UASAM)
}

\author{
Maria Garcia-Fernandez ${ }^{1}$, Yuri Alvarez-Lopez ${ }^{1}$, Fernando Las Heras ${ }^{1}$, Ana Arboleya-Arboleya ${ }^{2}$, \\ Borja Gonzalez-Valdes ${ }^{3}$, Yolanda Rodriguez-Vaqueiro ${ }^{3}$ \\ ${ }^{1}$ Area of Signal Theory and Communications, University of Oviedo, Gijón, Spain, mariagarcia@tsc.uniovi.es \\ ${ }^{2}$ Dept. of Signal Theory and Communications, University Rey Juan Carlos, Madrid, Spain \\ ${ }^{3}$ AtlantTIC Research Centre, University of Vigo, Vigo, Spain
}

\begin{abstract}
The main goal of this contribution is to provide an overview of an Unmanned Aerial System for Antenna Measurement (UASAM), which will be presented at the AMTA Scientific Workshop on "UAV-based Antenna and Field Measurements". UASAM is a compact low-cost system that can be used for in-situ antenna measurement and diagnostics. It makes use of a Real Time Kinematik (RTK) and a laser altimeter to georefer the measurements with $\mathrm{cm}$-level accuracy. Field radiated by the Antenna Under Test (AUT) is measured with a low-cost power detector at several acquisition surfaces (even non-canonical surfaces) around the AUT (in the near field region). Next, an iterative phase retrieval technique is applied, being able to recover the AUT aperture fields. Furthermore, from these fields, Near-Field to Far-Field (NF-FF) transformation can be used to retrieve the radiation pattern. Several application examples for different kinds of AUTs will be shown in the workshop. In addition, the impact of positioning and geo-referring accuracy will be discussed as well. This system is of great interest for those industrial applications where antenna diagnostics and radiation pattern assessment capabilities are required (e.g. analysis of antenna arrays, tilt testing of base station antennas, etc.) avoiding the need of stopping operational conditions.
\end{abstract}

Index Terms-phaseless antenna measurement, antenna diagnostics, Unmanned Aerial Vehicles (UAVs), Near-Field to FarField transformation (NF-FF).

\section{INTRODUCTION}

In the last few years, there has been a great growth in the development of Unmanned Aerial Vehicles (UAVs). As a result, new emerging applications of UAVs are continuously proposed. Among these applications, several UAV-based systems have been presented for antenna measurement [1]. One of the main advantages of these systems is that they are able to measure the Antenna Under Test (AUT) in its operational location and thus, they take into account the influence of the surroundings. Therefore, these systems are of great interest for fast, low cost evaluation of the performance of the AUT (antenna diagnostics, radiation pattern) in those cases where high accurate measurements (e.g. those conducted at anechoic chambers) are not required.

UAV-based antenna measurement systems can be classified according to several criteria, such as the RF devices involved in the measurements or the region of operation (Near-Field, NF, or Far-Field, FF). Regarding the first criterion, the RF devices onboard the UAV are usually: a spectrum analyzer [2], a power detector [3] or a RF transmitter [4] (being the
AUT the receiver). Concerning the region of operation, most systems work at the FF region, measuring the antenna radiation pattern directly. For low frequency antennas, the FF region could be several hundreds of meters away from the AUT. Consequently, flight restrictions and UAVs flight autonomy might limit the practical application of these systems. To overcome these problems, we have proposed an Unmanned Aerial System for Antenna Measurement (UASAM) working in the NF region [5][6].

UASAM is a low cost, compact and low complexity system that makes use of a power detector onboard the UAV. Due to the lack of phase information, a phase retrieval technique called phaseless Sources Reconstruction Method (pSRM) is used to recover the AUT aperture fields, providing antenna diagnostics information. From these fields, a NF-FF transformation is used to obtain the radiation pattern. UASAM has been tested for antenna measurements and diagnostics up to $\mathrm{C}$ band. Furthermore, its performance has been evaluated comparing the results with those obtained with anechoic chamber measurements.

\section{DESCRIPTION OF UASAM}

An overview of the proposed system is shown in Fig. 1. The UAV follows a predefined flight plan (set using waypoints), which describes the measurement grid. It sends the georeferred amplitude-only measurements to a ground station, where they are post-processed to obtain the AUT aperture fields and the radiation pattern.

A picture of the UASAM prototype is shown in Fig. 2. UASAM is mainly composed by the following subsystems:

- UAV (Fig. 2, green color) including the flight controller, the communication systems and the common positioning sensors (Inertial Measurement Units, barometer and Global Navigation Satellite Systems, GNSS).

- Accurate positioning subsystem (Fig. 2, blue color): Real Time Kinematic (RTK) system and laser rangefinder. The RTK system is composed by two RTK beacons: one onboard the UAV and another one on the ground working as a base station. The latter estimates the corrections that must be applied to the GNSS signals and forwards them to the beacon onboard the UAV. 


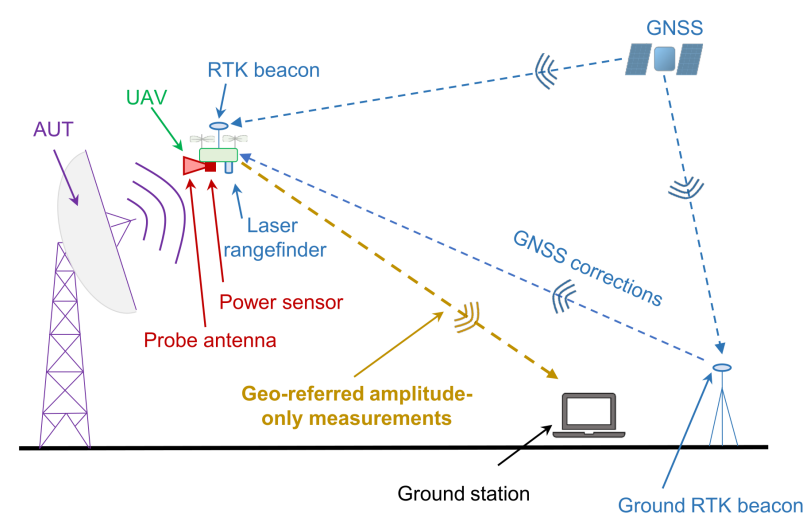

Fig. 1. Overview of the UASAM.

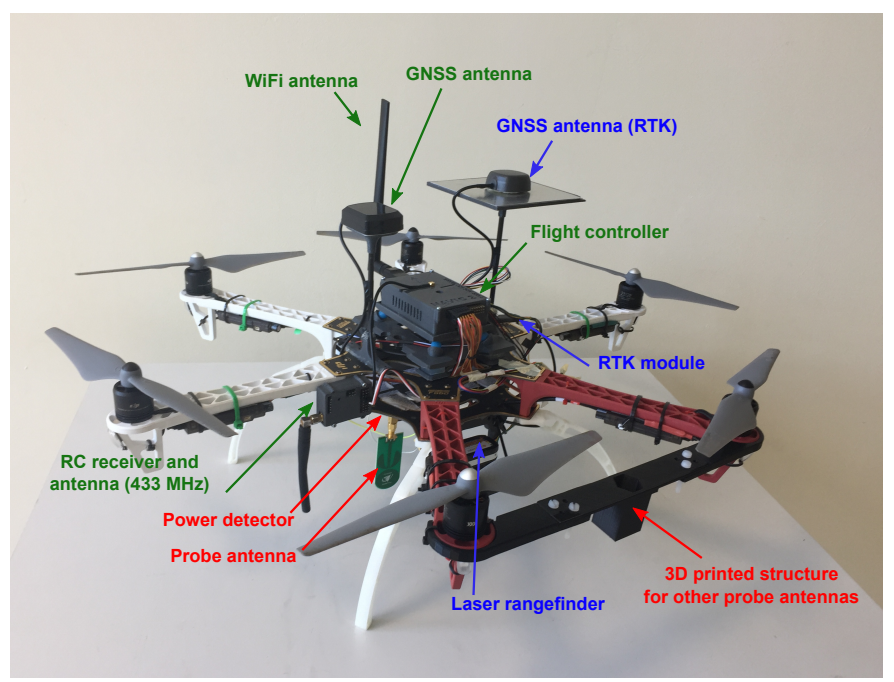

Fig. 2. UASAM prototype.

- Antenna measurement subsystem (Fig. 2, red color): power detector and probe antenna.

- Ground control station.

\section{METHODOLOGY}

As mentioned before, due to the use of a power detector instead of a coherent receiver, phase information is not available. Therefore, a phase retrieval technique called pSRM [7] is used to obtain an equivalent electric and magnetic currents distribution on the AUT aperture. Then, this distribution is used to perform antenna diagnostics and to calculate the radiation pattern (by means of NF-FF transformation techniques).

This technique has also been used for measuring antennas working at $865-868 \mathrm{MHz}$ frequency band using passive RFID (Radio Frequency IDentification) tags onboard a UAV and a RFID reader connected to the AUT [8].

The main advantage of the iterative phase retrieval technique based on the pSRM is that the measurement points can be arbitrarily distributed (as long as a quarter wavelength sampling rate is fulfilled). To ensure that the phase is properly recovered,

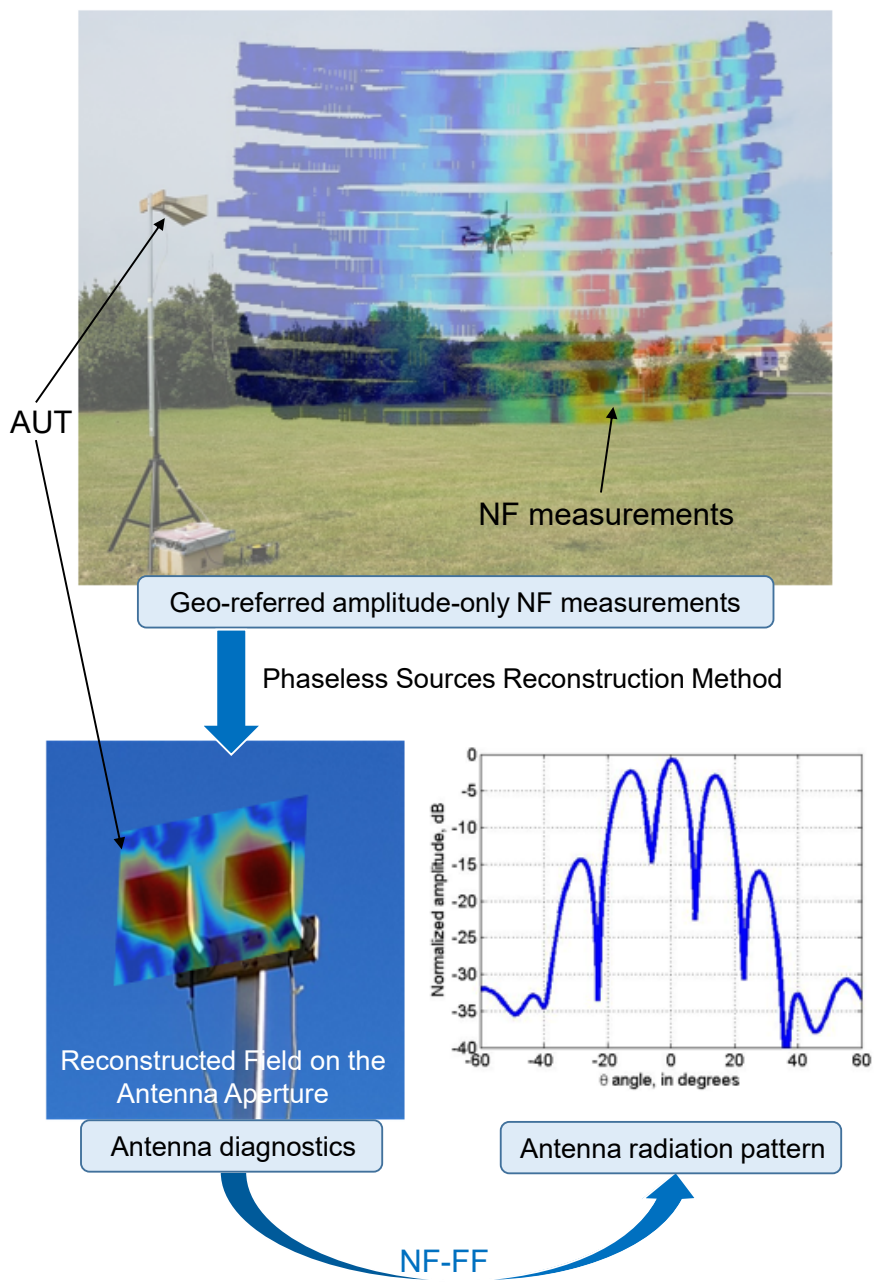

Fig. 3. Workflow of the post-processing steps for a two-horn antenna array working at $4.6 \mathrm{GHz}$.

amplitude-only measurements must be acquired in at least two different acquisition surfaces. Several acquisition surfaces, such as two concentric cylinders or two parallel planes have been considered aiming to test UASAM performance in terms of measurement accuracy and acquisition time.

\section{RESUlts}

A workflow of the post-processing steps for one application example is shown in Fig. 3. In this example a two-horn antenna array working at $4.6 \mathrm{GHz}$ is measured. The measurement grid is composed by two concentric half-cylindrical surfaces. The measurements acquired with the power detector at one of those surfaces is depicted in the upper part. These geo-referred amplitude-only NF measurements are post-processed with pSRM to reconstruct the field on the antenna aperture, which could be used to detect malfunctioning elements. Finally, with a NF-FF transformation, the radiation pattern is retrieved. 


\section{CONCLUSION}

In this contribution, an overview of an Unmanned Aerial System for Antenna Measurement (UASAM) has been presented. Thanks to the use of a geo-referring system that provides cm-level accuracy, UASAM is capable of performing measurements in the NF region of the AUT. Amplitude-only measurements acquired with a simple power detector are postprocessed by means of an iterative phase retrieval technique to recover the fields on the antenna aperture, later applying NF-FF transformation to calculate the radiation pattern. These features result in a versatile system that provides in-situ full antenna diagnostics and characterization. UASAM can be used for the evaluation of mobile networks base station antennas performance, the detection of large reflector antenna distortion, or the location of malfunctioning elements in large array antennas, among others.

\section{ACKNOWLEDGMENT}

This work has been partially supported by the "Ministerio de Economía y Competitividad" of Spain/FEDER under projects TEC2014-55290-JIN (PORTEMVISION) and TEC2014-54005-P (MIRIIEM); by the "Ministerio of Educación y Cultura" of Spain under FPU grant FPU15/06341; and by the Government of Asturias under project GRUPIN 14-114.

\section{REFERENCES}

[1] T. Fritzel, R. Strauss, H.-J. Steiner, C. Eisner, T. Eibert, "Introduction into an UAV-based Near-Field system for in-situ and large-scale antenna measurements," in Proc. of the IEEE Conference on Antenna Measurements and Applications (CAMA), Syracuse (USA), October 2016.

[2] J. Schreiber, "Antenna pattern reconstitution using unmanned aerial vehicles (UAVs)," in Proc. of the IEEE Conference on Antenna Measurements and Applications (CAMA), Syracuse (USA), October 2016.

[3] M. S. Sharawi, "Unmanned aerial vehicle for antenna radiation characterization,” US Patent Appl. US 20160088498 A1, published March 2016.

[4] G. Virone, F. Paonessa, E. Capello, O. A. Peverini, et al., "UAVbased antenna and field measurements," in IEEE Conference on Antenna Measurements and Applications (CAMA), Syracuse (USA), October 2016.

[5] M. Garcia-Fernandez, Y. Alvarez-Lopez, A. Arboleya, B. GonzalezValdes, Y. Rodriguez-Vaqueiro, M. E. de Cos, F. Las Heras, "Antenna Diagnostics and Characterization using Unmanned Aerial Vehicles," IEEE Access, vol. 5, pp. 23563-23575, September 2017.

[6] Y. Alvarez-Lopez, M. Garcia-Fernandez, F. Las Heras, A. ArboleyaArboleya, B. Gonzalez-Valdes, Y. Rodriguez-Vaqueiro, A. Garcia-Pino, "Sistema aerotransportado y método para la caracterización y medida de antenas o sistemas radiantes," Spanish Patent Application P201700209, PCT 15-03-2017.

[7] Y. Alvarez, F. Las-Heras, M. R. Pino, "The sources reconstruction method for amplitude-only field measurements," IEEE Transactions on Antennas and Propagation, vol. 58, no. 8, pp. 2776-2781, Aug. 2010.

[8] Y. Alvarez-Lopez, M. Garcia-Fernandez, G. Alvarez-Narciandi, A. Arboleya-Arboleya, F. Las-Heras, S. G. Cortes, M. F. Cabanas, "In situ antenna diagnostics and characterization system based on RFID and Remotely Piloted Aircrafts," Sensors and Actuators A, vol. 269, pp. 2940, 2018. 